\title{
ANÁLISE HISTOMORFOMÉTRICA DA LASERTERAPIA DE BAIXA INTENSIDADE EM FERIDAS CIRÚRGICAS NA PELE DE RATOS
}

\author{
HISTOMORPHOMETRIC ANALYSIS OF LOW-INTENSITY LASER \\ THERAPY IN SURGICAL WOUNDS IN RAT SKIN
}

\author{
Lucinéia Pereira de Jesus*; Caroline Bittencourt"; Kim Guimarães Caçula**; \\ Raul Pallotta Filho M.Sc.***; Lucio Frigo Ph.D.***; \\ Walter João Genovese,Ph.D. ${ }^{* * *}$; Maria Teresa Boti Rodrigues Santos Ph.D.*** \\ * Cirurgião-Dentista \\ ** Biólogo \\ ${ }^{* * *}$ Professor do curso de Odontologia da Universidade Cruzeiro do Sul. \\ E-mail: luciofrigo@uol.com.br \\ Recebido para publicação em 08/10/2010 \\ Aceito para publicação em 22/11/2010
}

\begin{abstract}
RESUMO
O objetivo deste estudo foi avaliar qualitativamente e quantitativamente o efeito de dois lasers, com diferentes comprimentos de onda, no processo inflamatório induzido por ferida cirúrgica na região dorsal de ratos. Foram utilizados 18 ratos Rattus novergicus, machos, pesando entre 200 e $250 \mathrm{~g}$, nos quais foi realizada uma incisão cirúrgica de $0,5 \mathrm{~cm}$ de extensão. Após a incisão, o Grupo $\mathrm{I}(\mathrm{n}=6)$ foi submetido à irradiação de forma puntiforme com laser de AsGa-Al ECCO Fibras ${ }^{\circledR}$ (Ecco Fibras ópticas e Dispositivos Ltda.), $50 \mathrm{~mW}$ de potência, comprimento de onda de $660 \mathrm{~nm}$, distância focal de $1 \mathrm{~cm}$ e densidade energética de $3 \mathrm{~J} / \mathrm{cm}^{2}$. O Grupo II $(\mathrm{n}=6)$ foi irradiado com laser AsGa VISION® (Laser-und Medizin-Technologie, Berlin) com programação para laserterapia de baixa intensidade, potência de $4 \mathrm{~W} / \mathrm{cm}^{2}$, comprimento de onda $980 \mathrm{nn}$, distância focal de $1 \mathrm{~cm}$ e densidade energética de $12 \mathrm{~J} / \mathrm{cm}^{2}$. O grupo III não foi irradiado pós incisão cirúrgica. O protocolo de aplicação da laserterapia foi no ato cirúrgico, 24, 48 horas. O teste não-paramétrico de Kruskall-Wallis foi utilizado para a análise estatística dos resultados $(\mathrm{p}<0,05)$, comparando os grupos I, II e III nos tempos 24 e $72 \mathrm{~h}$. Os resultados mostraram redução estatisticamente significante $(\mathrm{p}=0,0236)$ para o número de macrófagos no Grupo I no período de $24 \mathrm{~h}$. O número de fibroblastos foi significantemente maior $(\mathrm{p}=0,0067)$ para o Grupo III no período de $24 \mathrm{~h}$. O Grupo I apresentou quantidade de fibras colágenas do tipo I significantemente menor $(p<0,001)$ que os demais Grupos no período de $24 \mathrm{~h}$ e o Grupo III apresentou crescimento significativo dessas fibras em relação aos outros Grupos no período de $72 \mathrm{~h}(\mathrm{p}<0,001)$. Com relação às fibras colágenas do tipo III, o Grupo I apresentou aumento significante $(p<0,001)$ em relação aos outros Grupos no período de $24 \mathrm{~h}$, porém no de $72 \mathrm{~h}$ foi o Grupo II que apresentou quantidade significantemente maior $(\mathrm{p}<0,001)$. A laserterapia de baixa intensidade tanto com $\lambda$ $=660 \mathrm{~nm}$, como $\operatorname{com} \lambda=980 \mathrm{~nm}$, mostrou aspectos macroscópicos e microscópicos
\end{abstract}


qualitativos e quantitativos que indicam a atenuação do processo inflamatório e aceleração do processo reparativo no modelo experimental utilizado.

Palavras-Chave: Lasers. Inflamação. Fibroblastos. Fibrócitos. Macrófagos. Neutrófilos. Colágeno.

\begin{abstract}
The aim of this study was to evaluate qualitatively and quantitatively the effect of two lasers with different wavelengths in the inflammatory process induced by surgical wounds in the dorsal region of rats. For the study, 18 Rattus norvegicus male rats weighing between 200 and $250 \mathrm{~g}$ were used; each rat suffered a surgical incision of $0.5 \mathrm{~cm}$ in length. After the incision, Group $\mathrm{I}(\mathrm{n}=6)$ was subjected to irradiation with a punctiform laser GaAs-Al ECCO ${ }^{\circledR}$ fibers (optical fibers and devices Ecco Ltda.) $50 \mathrm{~mW}$ of power, wavelength of $660 \mathrm{~nm}$, distance $1 \mathrm{~cm}$ and focal energy density $3 \mathrm{~J} /$ $\mathrm{cm} 2$. Group II $(\mathrm{n}=6)$ was irradiated with GaAs VISION ${ }^{\circledR}$ (Laser-und MedizinTechnologie, Berlin) programmed for low-intensity laser therapy, power $4 \mathrm{~W} / \mathrm{cm} 2$, $980 \mathrm{nn}$ wavelength, focal length and density of $1 \mathrm{~cm} 12 \mathrm{~J} / \mathrm{cm} 2$ of energy. The third group was not irradiated after the surgical incision. The protocol for the application of laser therapy was in surgery, 24 hours, 48 hours. The non-parametric KruskalWallis test was used for statistical analysis $(p<0.05)$ comparing groups I, II and III after 24 and 72 hours. The results showed a statistically significant reduction $(p=0.0236)$ in the number of macrophages in Group I in the 24-hour period. The number of fibroblasts was significant higher $(p=0,0067)$ in Group III in the 24-hour period. Group I presented a smaller amount of collagen type I significantly lower ( $p<0.001)$ than the other groups in the 24-hour period and, Group III showed a significant growth of these fibers in relation to the other groups in the period of 72 hours $(p<0.001)$. With respect to collagen type III, Group I showed a significant increase $(p<0.001)$ compared with the other groups in the 24-hour period, but in the 72-hour period, Group II showed a significant greater amount $(p<0.001)$. Lowintensity laser therapy with both $\lambda=660 \mathrm{~nm}$ and $\lambda=980 \mathrm{~nm}$ showed macroscopic and microscopic as well as qualitative and quantitative aspects that indicate the attenuation of the inflammatory process and the acceleration of the repair process in this experimental model.

Keywords: Lasers. Inflammation. Fibroblasts. Fibrocytes. Macrophages. Neutrophils. Collagen.
\end{abstract}

\section{Introdução}

A inflamação é uma seqüência complexa de eventos celulares que objetiva o restabelecimento das condições homeostáticas anteriores a lesão. Os fenômenos celulares envolvem alterações hemodinâmicas de fluxo e permeabilidade vascular; ativação e transmigração de leucócitos que desempenham papel central no processo com produção de fatores solúveis, realização de fagocitose desencadeando a resposta imunológica. Entre os fatores solúveis produzidos pelos leucócitos encontramos aqueles responsáveis pela multiplicação celular que é imprescindível para o retorno à condição anterior a lesão, ou seja, o processo de reparação.

Embora separados temporalmente, a inflamação e a reparação são fenômenos indissociáveis, que se influenciam mutuamente. A terapêutica moderna dispõe de um grande arsenal para a modulação do processo inflamatório, entre os mais recentes, podemos citar a laserterapia de baixa intensidade.

A ação terapêutica da energia do laser baseia-se nas propriedades da interação com os tecidos. Dependendo do comprimento de onda da luz emitida a absorção específica da energia depositada pode ativar estruturas intra ou extracelulares. 
As primeiras aplicações de laser de baixa intensidade na modulação de processo inflamatório/ reparatório datam da década de 60 com os relatos clínicos de Mester et al. (1968). Evidências clínicas apontam para uma melhora significativa em alguns sinais e sintomas do processo inflamatório com redução de dor e edema, além da aceleração do processo reparatório como um todo. (MEYERS, 1990).

A terapia com laser também é apontada, em modelos experimentais, como agente redutor do volume de edema no processo inflamatório em tecidos irradiados, além de promover efeitos analgésicos pela inibição da sensibilização dos nocireptores, envolvendo mediadores hiperalgésicos ao invés de receptores opióides periféricos (FERREIRA et al., 2005).

No que concerne à reparação, a laserterapia tem sido utilizada na clínica com grande eficácia no controle lesões traumáticas em indivíduos com grande espasticidade em musculatura mastigatória (MOREIRA et al., 2004) e úlceras em pés de diabéticos (MAIYA; KUMAR; RAO, 2005).

O emprego da laserterapia de baixa intensidade, ou terapia com luz monocromática de diodos, tem se tornado popular e vem sendo utilizado em várias condições clínicas, incluindo a promoção de reparação de feridas (NUNEZ et al., 2004). Esse processo é positivamente afetado quando os parâmetros da laserterapia são corretamente utilizados (DO NASCIMENTO et al., 2004).

A liberação de fatores de crescimento dos fibroblastos pode acelerar a reparação tecidual facilitando o processo reparacional, quando a irradiação com laser é feita em comprimentos de onda adequados (MAIYA; KUMAR; RAO, 2005).

A proliferação das células endoteliais é importante no processo de reparação tecidual e a irradiação nos tecidos com laser de baixa intensidade influência a proliferação dessas células, contribuindo para o aumento da angiogênese e conseqüente aporte sanguíneo, acelerando o processo reparativo (SCHINDL et al., 2003).

Dentre os efeitos também observados pela estimulação da irradiação com laser sobre os tecidos lesados, encontra-se a ativação dos canais ATP-sensitivos, promovendo facilitação dos efeitos reparativos (KOLPAKOVA et al., 2003).
O efeito da laserterapia na fase inicial da reparação atua aumentando a atividade fagocitária dos macrófagos, facilitando e estabelecendo condições necessárias para o início da fase proliferativa (WALSH, 1997).

\section{Objetivo}

O objetivo deste estudo foi avaliar qualitativamente e quantitativamente o efeito de dois lasers, com diferentes comprimentos de onda, no processo inflamatório induzido por ferida cirúrgica na região dorsal de ratos.

\section{Metodologia}

Após a aprovação do protocolo deste estudo pelo Comitê de Ética em Pesquisa da Universidade Cruzeiro do Sul (CEP 058/05), foram utilizados 18 Rattus novergicus, machos, pesando entre 200 e $250 \mathrm{~g}$ fornecidos pelo biotério da UNICSUL, onde foram mantidos em regime de claro/escuro a livre curso e alimentação ad libitum.

Os animais foram divididos em 3 grupos de 6 animais aleatoriamente.

Grupo I - Os animais foram anestesiados com $0,4 \mathrm{ml}$ de solução de Francotar ${ }^{\circledR}$ (Agribrands do Brasil Ltda) e Rompun ${ }^{\circledR}$ (Agribrands do Brasil Ltda) 1:1 por $100 \mathrm{~g}$ peso animal, por via intramuscular, seguindo-se tricotomia na região dorsal dos animais, e imediatamente após, foi realizada uma incisão de $0,5 \mathrm{~cm}$ com lâmina de bisturi número 15 (Med blade ${ }^{\circledR}$ ). Em seguida, foi feita a irradiação de forma puntiforme com laser de AsGa ECCO Fibras ${ }^{\circledR}$ (Ecco Fibras ópticas e Dispositivos Ltda.), $50 \mathrm{~mW}$ de potência, comprimento de onda de 660 $\mathrm{nm}$, distância focal de $1 \mathrm{~cm}$ e densidade energética de $3 \mathrm{~J} / \mathrm{cm}^{2}$. O protocolo terapêutico foi imediato, 24 e 48 horas após a lesão.

Grupo II - Os animais foram submetidos à mesma seqüência de procedimentos que o Grupo I, com exceção ao comprimento de onda e marca comercial do laser InGa VISION ${ }^{\circledR}$ (Laser-und Medizin-Technologie, Berlin) com programação 
para laserterapia de baixa intensidade, potência de $4 \mathrm{~W} / \mathrm{cm}^{2}$, comprimento de onda $980 \mathrm{nn}$, distância focal de $1 \mathrm{~cm}$ e densidade energética de $12 \mathrm{~J} / \mathrm{cm}^{2}$. Foi realizado o mesmo regime protocolo terapêutico.

Grupo III - Os animais foram submetidos à mesma seqüência que os Grupos I e II, entretanto não foram irradiados. Este foi considerado como Grupo Controle.

Todos os animais foram acomodados em gaiolas individuais, em temperatura ambiente (cerca de $25^{\circ} \mathrm{C}$ ).

Depois de completada a seqüência experimental proposta, três animais de cada grupo foram anestesiados com doses letais dos mesmos anestésicos já citados, e sacrificados em $24 \mathrm{~h}$. Os outros nove animais foram sacrificados em $72 \mathrm{~h}$ após a lesão cirúrgica.

A seguir, foram extraídos os tecidos onde foram feitas as lesões e a laserterapia. As peças assim obtidas foram fixadas em solução de formol a 4\% durante 24 horas, sendo posteriormente desidratadas, diafanizadas e incluídas em parafina, orientadas de tal forma, a permitir cortes no sentido paralelo ao longo eixo da ferida cirúrgica, sendo feitas três lâminas para cada animal.

Foram realizados cortes semi-seriados com $5 \mu \mathrm{m}$ de espessura. A contagem dos tipos celulares (neutrófilos, macrófagos, fibroblastos e fibrócitos), bem como a quantificação das fibras de colágeno (tipo I e III) foi realizada com um disco de integração de 100 pontos, acoplado à ocular de $12,5 \mathrm{x}$ de aumento e usando objetiva de $100 \mathrm{x}$ de aumento (imersão). Foram contados 5 campos microscópicos de cada corte dos existentes em cada lâmina, corados em Hematoxilina e Eosina (H.E.)

$\mathrm{O}$ teste não-paramétrico de Kruskall-Wallis foi utilizado para a análise estatística dos resultados $(p<0,05)$, comparando os grupos I, II e III nos tempos 24 e $72 \mathrm{~h}$.

\section{Resultados}

A análise dos cortes histológicos mostrou diferenças marcantes nas características do processo inflamatório/reparativo dos diferentes grupos.

\section{Análise qualitativa do processo inflamatório/reparativo}

A análise das características macroscópicas evolutivas do processo inflamatório/reparativo mostrou nos grupos submetidos à laserterapia (Grupos I e II) atenuação dos sinais inflamatórios relativos à menor edema e rubor, bem como aproximação maior e mais rápida das bordas da ferida em relação ao Grupo Controle.

A análise dos cortes histológicos de $24 \mathrm{~h}$ do Grupo III mostrou que o epitélio apresentava deiscência com a presença de um coágulo bem evidente cercado por células inflamatórias. Situação semelhante foi observada nos demais grupos, sendo que, no Grupo II o infiltrado inflamatório peri-coágulo apresentava-se bastante exuberante.

A análise das lâminas de $72 \mathrm{~h}$ mostrou um quadro diverso. No Grupo Controle pode-se observar ainda a presença da deiscência epitelial e o coágulo interposto, porém, as bordas do tecido epitelial estavam dilatadas, com grande quantidade de células, sendo que algumas já se projetavam por baixo do coágulo em direção a extremidade oposta.

No Grupo I o tecido epitelial já havia alcançado o lado oposto, não havendo deiscência. O epitélio apresentava 3 ou 4 camadas de células, com uma definição bastante clara do estrato basal. Também era freqüente a observação de figuras de mitose.

No grupo II o tecido epitelial também havia alcançado o lado oposto, porém, apresentava algumas regiões de adelgaçamento com somente 1 ou 2 camadas de células sem definição clara dos estratos.

\section{Análise quantitativa do processo inflamatório/reparativo}

O número de neutrófilos dos Grupos I, II e III não apresentou diferença estatisticamente significante nos períodos de $24(\mathrm{p}=0,9874)$ e $72 \mathrm{~h}$ $(\mathrm{p}=0,1856)$. (Gráfico 1). 
Gráfico 1 - Contagem de neutrófilos nos Grupos I (Ecco), II (Vision) e III (Controle) após laserterapia de baixa intensidade em 24 e $72 \mathrm{~h}$.

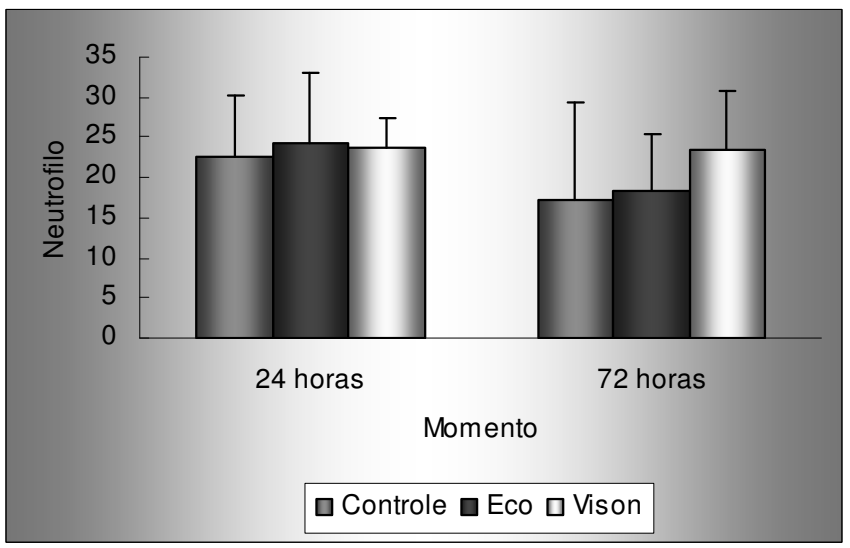

Entretanto o número de macrófagos apresentou redução estatisticamente significante $(\mathrm{p}=0,0236)$ para o Grupo I (Ecco) em relação ao Grupo II (Vision) no período de $24 \mathrm{~h}$. Para o período de $72 \mathrm{~h}$ não houve diferença significante entre os grupos $(\mathrm{p}=0,0617)$. (Gráfico 2).

Gráfico 2 - Contagem de macrófagos nos Grupos I (Ecco), II (Vision) e III (Controle) após laserterapia de baixa intensidade em 24 e $72 \mathrm{~h}$.

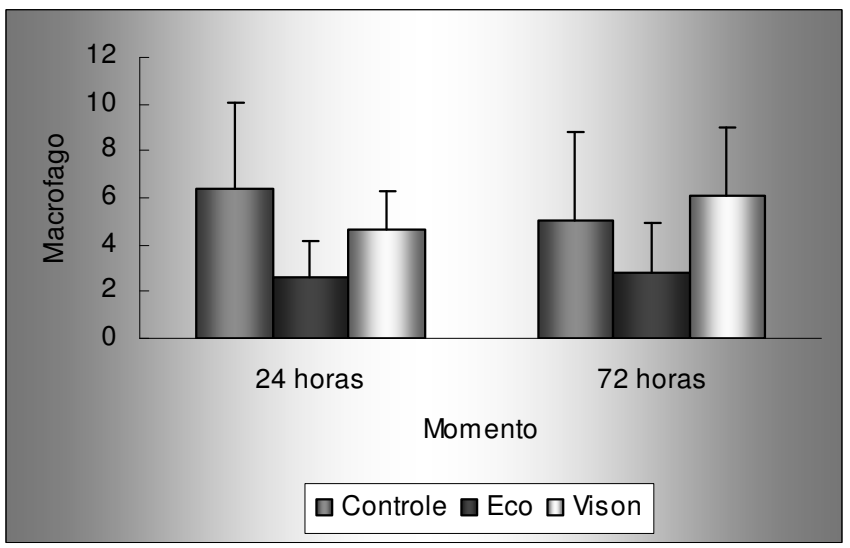

O número de fibroblastos mostrou-se significativamente maior no Grupo III (Controle) em relação ao Grupo I (Ecco) no período de $24 \mathrm{~h}$ $(\mathrm{p}=0,0067)$; já para o período de $72 \mathrm{~h}$ não houve diferença estatística $(p=0,0580)$ entre os grupos. (Gráfico 3).
Gráfico 3 - Contagem de fibroblastos nos Grupos I (Ecco), II (Vision) e III (Controle) após laserterapia de baixa intensidade em 24 e $72 \mathrm{~h}$.

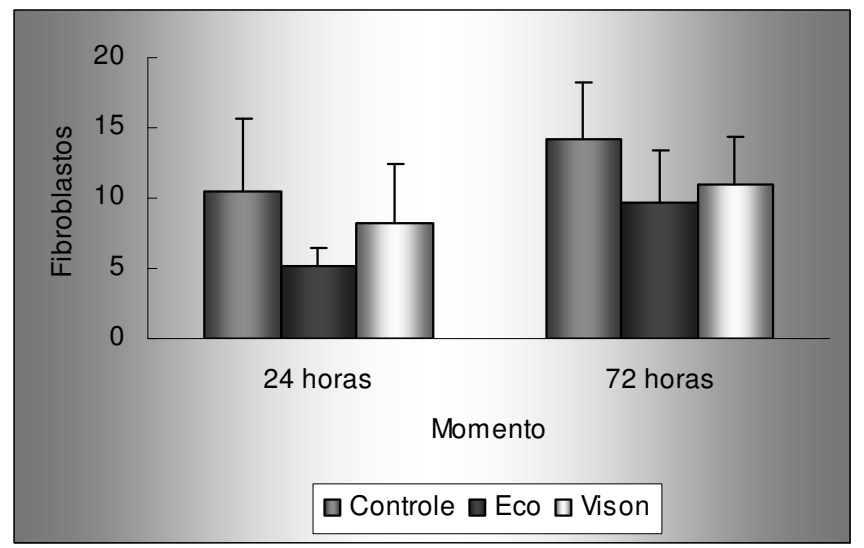

Os resultados não mostraram diferença significante para o número de fibrócitos entre os grupos nos períodos de $24(\mathrm{p}=0,0632)$ e $72 \mathrm{~h}(\mathrm{p}=0,3920)$ (Gráfico 4).

Gráfico 4 - Contagem de fibrócitos nos Grupos I (Ecco), II (Vision) e III (Controle) após laserterapia de baixa intensidade em $24 \mathrm{e} 72 \mathrm{~h}$.

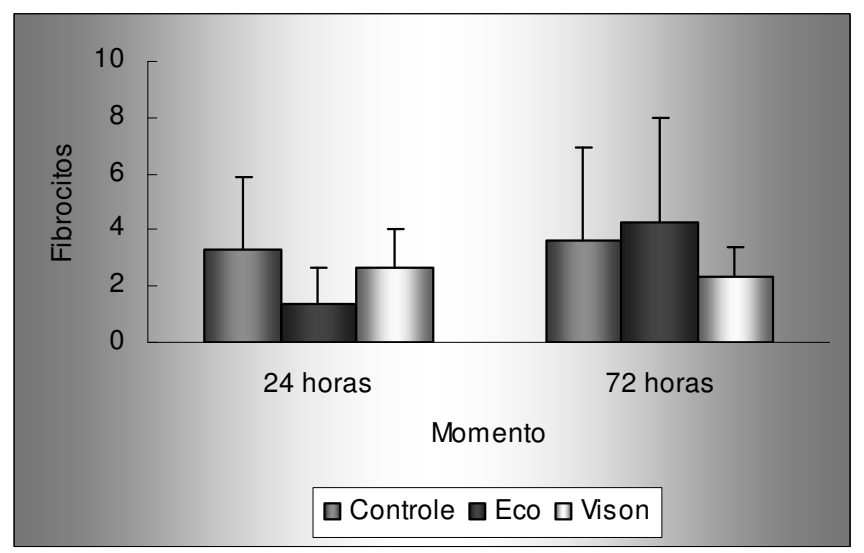

Entretanto o Grupo I (Eco) apresentou quantidade de fibras colágenas do tipo I significantemente menor comparado aos demais Grupos no período de $24 \mathrm{~h}(\mathrm{p}<0,001)$. Já para o período de $72 \mathrm{~h}$, o Grupo III (Controle) apresentou um crescimento significativo destas fibras, em relação aos demais grupos $(\mathrm{p}<0,001)$. (Gráfico 5). 
Gráfico 5 - Quantificação das fibras de colágeno I nos grupos I (Ecco), II (Vision) e III (Controle) após laserterapia de baixa intensidade em 24 e $72 \mathrm{~h}$.

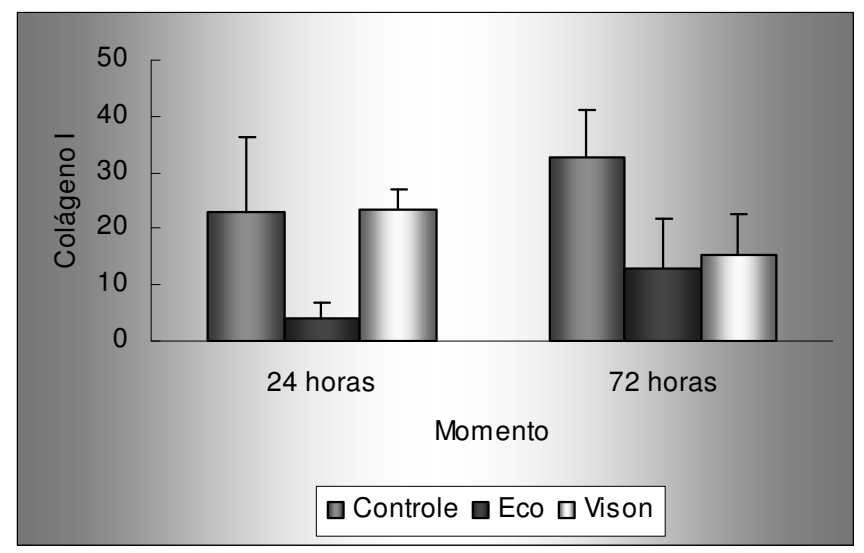

Para as fibras colágenas do tipo III o Grupo I (Ecco) apresentou número significativamente maior em relação aos demais Grupos no período de $24 \mathrm{~h}$ $(\mathrm{p}<0,001)$. No período de $72 \mathrm{~h}$ foi o Grupo II (Vision) que apresentou quantidade significativamente maior que os demais ( $\mathrm{p}<0,001)$. (Gráfico 6).

Gráfico 6 - Quantificação das fibras de colágeno III nos Grupos I (Ecco), II (Vision) e III (Controle) após laserterapia de baixa intensidade em 24 e $72 \mathrm{~h}$.

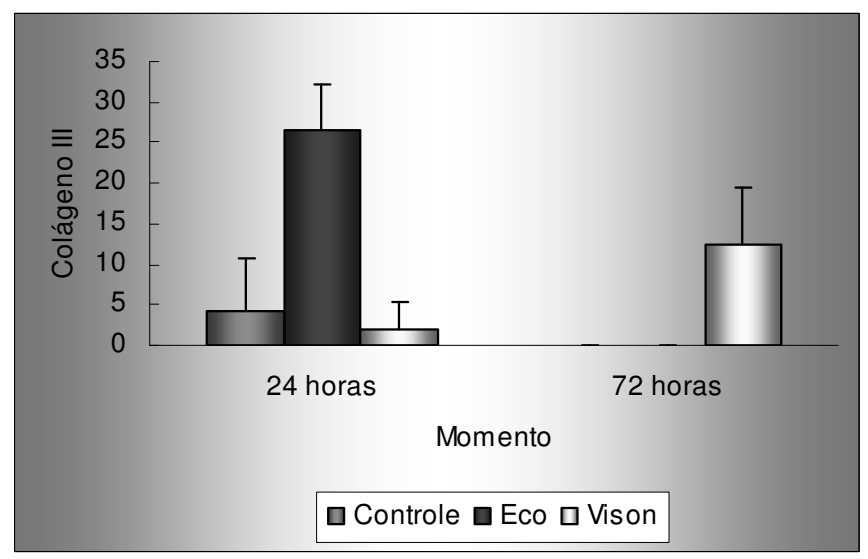

\section{Discussão}

A laserterapia de baixa intensidade é considerada um importante agente terapêutico coadjuvante no alívio dos sintomas pós-operatórios e na otimização dos processos reparativos subseqüentes.

Entretanto, para alcançar este fim é importante a observação dos corretos regimes terapêuticos e da dosimetria, como apontado por do Nascimento et al. (2004).

Os parâmetros para laserterapia são importantes a ponto de serem apontados como o principal agente causador de descrédito na comunidade médica, uma vez que, a inobservância destes leva a ineficácia da laserterapia, como apontado na meta-análise da literatura realizada por Lucas et al. (2002).

Evidências sobre o efeito modulatório da laserterapia de baixa intensidade em vários aspectos da inflamação, como edema, dor, permeabilidade vascular e peso do granuloma; podem ser encontradas na literatura (HONMURA et al.,1992; FERREIRA et al., 2005).

Estudos in vitro mostram que a laserterapia pode modular a estrutura e a função dos macrófagos, apontando uma potencialição da atividade fagocitária (KLEBANOV; POLTANOV; VLADIMIROV, 2003; DUBE; BANSAL; GUPTA, 2003). Nossos resultados mostram uma significativa diminuição do número de macrófagos nas primeiras $24 \mathrm{~h}$ após laserterapia com $660 \mathrm{~nm}$, o que é consistente com a constatação clínica de atenuação da inflamação. Achado semelhante foi relatado por Bayat et al. (2005). Além disso, Sakurai, Yamaguchi e Abiko (2000) descreveram uma diminuição da produção de prostaglandina E2 após laserterapia com laser de AsGa-Al, o que também aponta para a atenuação da inflamação observada na clínica.

A literatura é bastante rica no que concerne à aceleração do processo reparativo (BYRNES et al., 2004; PESSOA et al., 2004; MAIYA; KUMAR; RAO, 2005). Os estudos in vitro corroboram estes achados mostrando estímulo à proliferação dos fibroblastos (WEBB; DYSON, 2003). Assim, nossos achados relativos ao aumento do número de fibroblastos, após laserterapia com $660 \mathrm{~nm}$, nas primeiras $24 \mathrm{~h}$ estão de acordo com a literatura.

No tocante à produção de colágeno Yamamoto et al. (1996) e Pugliese et al. (2003) apontam um aumento na síntese de colágeno e elastina após laserterapia de baixa intensidade. Já Pereira et al. (2002) relatam a ineficácia da laserterapia no estímulo à síntese de colágeno. Embora tenhamos analisado o período de 24 e $72 \mathrm{~h}$, que não é o auge 
do processo reparativo, nossos resultados são ainda contrários aos descritos na literatura mostrando diminuição da quantidade de fibras colágenas do tipo I.

A literatura não apresenta qualquer relato sobre as fibras colágenas do tipo III, contudo, é importante ressaltar que este tipo de colágeno predomina no tecido de granulação, que é o primeiro passo no processo reparativo.

O estudo histomorfométrico de Damante et al (2004) realizado em gengiva de humanos não demonstrou diferença estatisticamente significante nos vários tipos celulares considerados, porém, a quantificação foi realizada em 7, 14, 21 e 60 dias após gengivoplastia.

No que concerne à comparação dos resultados obtidos com os dois diferentes tipos de laser utilizados, a primeira consideração deve ser feita em relação aos comprimentos de onda de cada aparelho: o Ecco Fibras (Grupo I) opera $\operatorname{com} \lambda=660 \mathrm{~nm}$ e o Vision Medizin (Grupo II) opera $\operatorname{com} \lambda=980 \mathrm{~nm}$.

A faixa de comprimento que abrange o vermelho até o infravermelho próximo (619 a 860nm) é a mais utilizada em laserterapia de baixa intensidade, esta seleção está baseada nos extensos trabalhos de Karu (1999), Karu, Piatibrat e Kalendo (1999), Karu (2004), Karu, Pyatibrat e, Afanasyeva (2005) e Karu e Kolyakov (2005) que demonstraram que em alguns picos desta faixa se encontram-se os efeitos celulares mais evidentes, como a absorção da energia pelas células, alteração da síntese de DNA e RNA, alteração da cinética de alguns transportadores de membrana e alteração do potencial oxidativo e redutor das mitocôndrias, bem como aumento da produção de ATP. A teoria vigente sobre o mecanismo de ação da laserterapia de baixa intensidade em nível celular está baseada nestes achados.

A energia do laser seria absorvida por moléculas específicas, denominadas cromóforos, sendo que as evidências apontam para a enzima citocrômo c oxidase da cadeia respiratória mitocondrial, desencadeando um desequilíbrio do potencial oxidativo/ redutor na referida organela, e aumento da síntese de ATP, como mostra a Figura 1.
Figura 1 - Esquema de uma célula mostrando o mecanismo de ação da laserterapia de baixa itensidade. Modificado de Karu (1999).

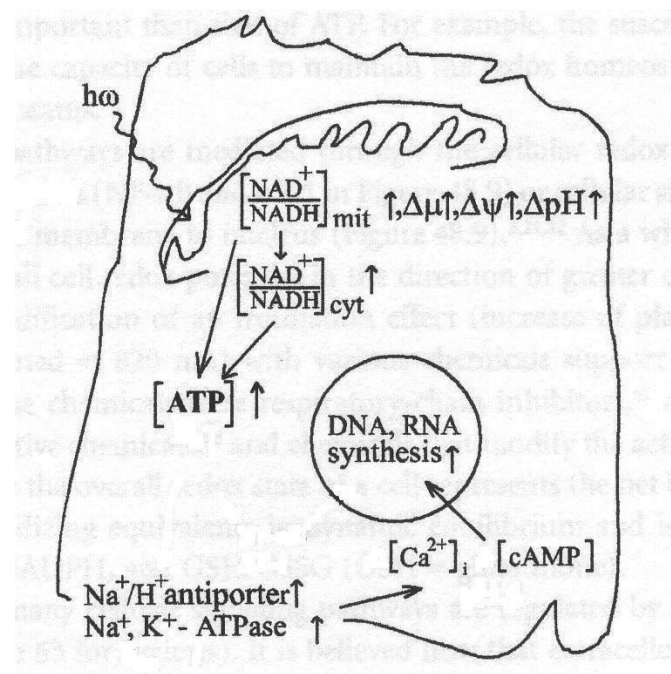

Por outro lado, poucas informações existem na literatura acerca da utilização e dos mecanismos da laserterapia de baixa intensidade $\operatorname{com} \lambda=880 \mathrm{~nm}$. O único relato deve-se a van Breugel e Bar (1992) que demonstram a absorção deste comprimento de onda por fibroblastos in vitro.

Nossos resultados mostram um significativo aumento da produção de colágeno III no Grupo irradiado $\operatorname{com} \lambda=980 \mathrm{~nm}$, em $72 \mathrm{~h}$. Podemos supor, assim, que a absorção deste comprimento de onda pelos fibroblastos pode desencadear a produção de colágeno tipo III.

\section{Conclusão}

A laserterapia de baixa intensidade tanto com $\lambda=660 \mathrm{~nm}$, como com $\lambda=980 \mathrm{~nm}$, mostrou aspectos macroscópicos e microscópicos qualitativos e quantitativos que indicam a atenuação do processo inflamatório e aceleração do processo reparativo no modelo experimental utilizado.

\section{REFERÊNCIAS}

BAYAT, M.; VASHEGHANI, M. M.; RAZAVI, N.; TAHERI, S.; RAKHSHAN, M. Effect of low-level laser therapy on the healing of second-degree burns in rats: a histological and microbiological study. J Photochem Photobiol B. Feb 1, v.78, n.2, p.171-7, 2005. 
BYRNES, K. R,; BARNA, L.; CHENAULT, V. M.; WAYNANT, R. W.; ILEV, I. K.; LONGO L, MIRACCO, C.; JOHNSON, B.; ANDERS, J. J. Photobiomodulation improves cutaneous wound healing in an animal model of type II diabetes. Photomed Laser Surg. Aug, v.22, n.4, p.281-90, 2004.

DAMANTE, C. A.; GREGHI, S. L.; SANT'ANA, A. C.; PASSANEZI, E.; TAGA, R. Histomorphometric study of the healing of human oral mucosa after gingivoplasty and low-level laser therapy. Lasers Surg Med., v.35, n.5, p.37784,2004

DO NASCIMENTO, P. M.; PINHEIRO, A. L.; SALGADO, M. A.; RAMALHO, L. M. A preliminary report on the effect of laser therapy on the healing of cutaneous surgical wounds as a consequence of an inversely proportional relationship between wavelength and intensity: histological study in rats. Photomed Laser Surg., Dec v.22, n.6, p.513-518, 2004.

DUBE, A.; BANSAL, H.; GUPTA, P. K. Modulation of macrophage structure and function by low level He-Ne laser irradiation. Photochem Photobiol Sci. Aug, v.2, n.8, p.851855, 2003.

FERREIRA, D. M.; ZANGARO, R. A.; VILLAVERDE, A. B.; CURY, Y.; FRIGO, L.; PICCOLO, G.; LONGO, I.; BARBOSA, D. G. Analgesic effect of He-Ne (632.8nm) low-level laser therapy on acute inflammatory pain. Photomed Laser Surg., Apr, v.23, n.2, p.177-181, 2005.

HONMURA, A.; YANASE, M.; OBATA, J.; HARUKI, E. Therapeutic effect of Ga-Al-As diode laser irradiation on experimentally induced inflammation in rats. Lasers Surg Med., v.2, n.4, p.441-449, 1992.

KARU, T. I. A suitable model for wound healing: how many times are we to stumble over the same block? Lasers Surg Med. 1999;25(4):283-284.

; PIATIBRAT, L. V.; KALENDO, G. S. Suppression of the intracellular concentration of ATP by irradiating with a laser pulse of wavelength lambda=820 nm. Dokl Akad Nauk., Jan, v.364, n.3, p.399-401,1999.

High-tech helps to estimate cellular mechanisms of low power laser therapy. Lasers Surg Med., v.34, n.4, p.298-299. 2004.

.; PYATIBRAT, L. V.; AFANASYEVA, N. I. Cellular effects of low power laser therapy can be mediated by nitric oxide. Lasers Surg Med., Apr, v.36, n.4, p.307-314, 2005.

; KOLYAKOV, S. F. Exact action spectra for cellular responses relevant to phototherapy. Photomed Laser Surg., Aug, v.23, n.4, p.355-361, 2005.

KLEBANOV, G. I.; POLTANOV, E. A.; VLADIMIROV, Iu A. Effect of low intensity laser light in the red range on macrophage superoxide dismutase activity. Biofizika, MayJun, v.48, n.3, p.462-473, 2003.
KOLPAKOVA, M. E.; VLASOV, T. D.; PETRISHCHEV, N. N.; VISLOBOKOV, A. I. Effect of the He-Ne laser irradiation on resistance of the isolated heart to the ischemic and reperfusion injury. Ross Fiziol Zh Im I M Sechenova, Dec; v.89, n.12, p.1496-1502, 2003.

LUCAS, C.; CRIENS-POUBLON, L. J.; COCKRELL, C. T.; DE HAAN, R. J. Wound healing in cell studies and animal model experiments by Low Level Laser Therapy; were clinical studies justified? a systematic review. Lasers Med Sci.; v.17, n.2, p.110-34, 2002.

MAIYA, G. A.; KUMAR, P.; RAO, L. Effect of low intensity helium-neon (He-Ne) laser irradiation on diabetic wound healing dynamics Photomed Laser Surg. Apr, v.23, n.2, p.187-90, 2005.

MESTER, E.; JUHASZ, J.; VARGA, P.; KARIKA, G. Lasers in clinical practice. Acta Chir Acad Sci Hung., v.9, n.3, p.349-57, 1968.

MEYERS, A. D. Lasers and wound healing. Arch Otolaryngol Head Neck Surg. Oct, v.116, n.10, p.1128, 1990.

MOREIRA, L. A.; SANTOS, M. T.; CAMPOS, V. F.; GENOVESE, W. J. Efficiency of laser therapy applied in labial traumatism of patients with spastic cerebral palsy. Braz Dent J.; v.15 Spec N.:SI, p.29-33, 2004.

NUNEZ, S. C.; NOGUEIRA, G. E.; RIBEIRO, M. S.; GARCEZ, A. S.; LAGE-MARQUES, J. L. He-Ne laser effects on blood microcirculation during wound healing: a method of in vivo study through laser Doppler flowmetry. Lasers Surg Med., v.35, n.5, p.363-8, 2004.

PEREIRA, A. N.; EDUARDO, C. DE P.; MATSON, E.; MARQUES, M. M. Effect of low-power laser irradiation on cell growth and procollagen synthesis of cultured fibroblasts. Lasers Surg Med, v.31, n.4, p.263-7, 2002.

PESSOA, E. S.; MELHADO, R. M.; THEODORO, L. H.; GARCIA, V. G. A histologic assessment of the influence of low-intensity laser therapy on wound healing in steroid-treated animals. Photomed Laser Surg., v.22, n.3, p.199-204, Jun. 2004.

PUGLIESE, L. S.; MEDRADO, A. P.; REIS, S. R.; ANDRADE, $Z$ de A. The influence of low-level laser therapy on biomodulation of collagen and elastic fibers. Pesqui Odontol Bras.,2003 Oct-Dec;v.17, n.4, p.307-13, Epub, Apr. 19. 2004

SAKURAI, Y.; YAMAGUCHI, M.; ABIKO, Y. Inhibitory effect of low-level laser irradiation on LPS-stimulated prostaglandin E2 production and cyclooxygenase-2 in human gingival fibroblasts. Eur J Oral Sci., v.108, n.1, p.29-34, Feb. 2000.

SCHINDL, A.; MERWALD, H.; SCHINDL, L.; KAUN, C.; WOJTA, J. Direct stimulatory effect of low-intensity $670 \mathrm{~nm}$ laser irradiation on human endothelial cell proliferation. Br J Dermatol., v.48, n.2, p.334-6, Feb. 2003. 
VAN BREUGEL, H. H.; BAR, P. R. Power density and exposure time of He-Ne laser irradiation are more important than total energy dose in photo-biomodulation of human fibroblasts in vitro. Lasers Surg Med., v.2, n.5, p.528-37, 1992. WALSH, L. J. The current status of low level laser therapy in dentistry. Part 2. Hard tissue applications. Aust Dent J., v.42, n.5, p.302-6, Oct 1997.

WEBB, C.; DYSON, M. The effect of $880 \mathrm{~nm}$ low level laser energy on human fibroblast cell numbers: a possible role in hypertrophic wound healing. J Photochem Photobiol B., v.70, n.1, p.39-44, Apr. 2003.

YAMAMOTO, Y.; KONO, T.; KOTANI, H.; KASAI, S.; MITO, M. Effect of low-power laser irradiation on procollagen synthesis in human fibroblasts. J Clin Laser Med Surg., v.14, n.3, p.129-32, Jun.1996. 